\title{
Echocardiography as an approach for canine cardiac disease diagnosis
}

\author{
P. Singh ${ }^{1}$, N. Singh ${ }^{2}$, S. K. Mahajan ${ }^{3}$ and T. Singh ${ }^{3}$
}

1. Veterinary Polytechnic \& Regional Research and Training Centre, Kaljharani, Bathinda, Punjab, India; 2. Department of Veterinary \& A.H. Extension Education, College of Veterinary Science, Guru Angad Dev Veterinary and Animal Sciences University (GADVASU), Ludhiana, Punjab, India; 3. Department of Veterinary Surgery \& Radiology, College of Veterinary Science, GADVASU, Ludhiana, Punjab, India.

Corresponding author: P. Singh, e-mail: parampalsingh46@gmail.com, NS: nav2312@yahoo.com, SKM: skmahajan73@yahoo.co.in, TS: tarunbir@gmail.com

Received: 10-07-2014, Revised: 09-10-2014, Accepted: 15-10-2014, Published online: 16-11-2014

doi: 10.14202/vetworld.2014.960-965. How to cite this article: Singh P, Singh N, Mahajan SK, Singh T (2014) Echocardiography as an approach for canine cardiac disease diagnosis, Veterinary World 7(11): 960-965.

\begin{abstract}
Aim: The aim of the study was to establish the methods for diagnosis various canine cardiac ailments using echocardiography.

Materials and Methods: M-mode, two-dimensional echocardiography and Doppler studies were performed on 10 cases. Dogs showing signs of cardiac ailment either clinically, radiographic or via electrocardiographic examination were selected for study. Right parasternal short axis view was used for echocardiographic measurements. Right parasternal long axis and left parasternal apical views were used for Doppler studies. Doppler studies were performed at the level of aortic valve and atrioventricular valves for semi quantitative diagnosis of regurgitation.
\end{abstract}

Results: Dogs were found affected with dilated cardiomyopathy (DCM) (n=5), pericardial effusion (PE) $(n=1)$, combined PE and DCM ( $n=2)$ and remaining two showed abnormality on radiographic or electrographically evaluation but were found out to be normal echocardiographically $(n=2)$.

Conclusion: Echocardiography is an effective tool for diagnosis of various heart ailments.

Keywords: dilated cardiomyopathy, doppler studies, echocardiography, M-mode, pericardial effusion, two dimensional.

\section{Introduction}

Echocardiography provides a wealth of data concerning cardiac morphology and function. It can provide a lot of helpful information, including the size and shape of the heart, its pumping capacity and the location and extent of any damage to its tissues. It is especially useful for assessing diseases of the heart valves. It not only allows doctors to evaluate the heart valves, but also can detect abnormalities in the pattern of blood flow, such as the backward flow of blood through partly closed heart valves, known as regurgitation. For many patients, echocardiography is the definitive diagnostic tool. Latest diagnostic techniques like cardiac catheterization, radioisotope imaging, blood pool scintigraphy, angiography or nuclear imaging are either invasive or expensive whereas this is non-hazardous, non-invasive and relatively inexpensive imaging technique.

A well performed study coalesce the findings of the physical examination, electrocardiogram, and radiographs into a clearly defined diagnosis on which treatment decisions can be based. Two-dimensional (2D) and M-mode echocardiography has been used to evaluate the normal, as well as pathological heart by various authors [1-4].

The aim of the present study was to establish the methods for accurate diagnosis of various canine cardiac ailments using 2D and M-mode echocardiography.

Copyright: The authors. This article is an open access article licensed under the terms of the Creative Commons Attributin License (http:// creative commons.org/licenses/by/2.0) which permits unrestricted use, distribution and reproduction in any medium, provided the work is properly cited.

\section{Materials and Methods}

\section{Ethical approval}

Not necessary. All the clinical cases under this research were examined and treated as per standard examination and treating procedures.

The present clinical research included 10 dogs with some cardiac ailment; those were presented at the small animal teaching hospital, Guru Angad Dev Veterinary and Animal Sciences University (GADVASU), Ludhiana for echocardiography. The dogs underwent a thorough physical, clinical, hematological, serum biochemistry, radiographic, and electrocardiographic examination. Left ventricular outflow image that include the aortic root, left ventricular outflow tract, left ventricular chamber, mitral valve and left atrium (LA) were studied from both right parasternal long and short axis view. A four chamber and five chambered view were obtained from left parasternal apical view. 2D and M-mode echocardiograms were recorded and analyzed in accordance with the recommendation of the American Society of echocardiography [5]. Measurements of the cardiac structures were made from the frozen M-mode images on the screen. The following measurements were obtained: Left ventricular internal dimension at end-systole (LVIDs) and end-diastole (LVIDd), left ventricular posterior wall thickness at end-systole and at end-diastole, interventricular septal thickness at end-systole and at end-diastole. Aortic root dimension at end-diastole (aorta ratio [AO]), left atrial dimension during ventricular systole (LA) were measured through 2D images. Colour Doppler was applied on right parasternal long axis view and left parastenal 
apical view, both at four chamber and five chamber view, to check whether any kind of regurgitation was present or not. All the measurements were done in accordance to previous authors [6].

\section{Result}

These subjects were grouped on the basis of diagnosis of the disease condition, dilated cardiomyopathy (DCM) $(n=5)$, pericardial effusions (PE) $(n=1)$, combined DCM and PE $(n=2)$, increase in one or other parameters $(n=5)$ and remaining two that showed abnormality radiographic on or electrocardiographic evaluation but were found out to be normal on echocardiography $(n=2)$.

\section{DCM}

DCM was observed in five dogs (males=3, females=2) distributed as three Labrador species and one each of Saint Bernard and Mongrel breeds. All the dogs had a history of lethargy, depression, weight loss with or without a history of coughing, ascites and subcutaneous edema. The average age of the group

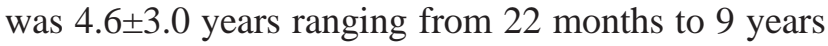
and with average body weight of $33.8 \pm 9.7$. Dogs had tachypnea with increased heart rate, and auscultation revealed heart murmurs in two cases with marked tachycardia. Lungs were found to be normal upon auscultation. The hemoglobin $(\mathrm{Hb})$ value was on the lower side, and white blood cell count was on a higher side with marked neutrophilia having mean values of $10.24 \pm 2.00(\mathrm{~g} / \mathrm{dL})$ and $22.30 \pm 16.35$ (103/cumm) respectively. The mean serum cholesterol level (mg/dL) of the dogs under study was 245.8 ranging from 190 to 296. Lateral and ventro-dorsal view of the chest revealed single chamber enlargement to generalized cardiomegaly with the heart covering more than $65 \%$ of the chest cavity. An increase in the sternal contact was seen with roundening of the heart in lateral view (Figure-1). Electrocardiogram of the dogs revealed tachycardia, arrhythmia, with changes like widening of P-wave (left atrial enlargement), change in shape of P-wave, increase in PR interval, flattening and merging of $\mathrm{T}$ and $\mathrm{P}$-wave, spiking of S-wave (right

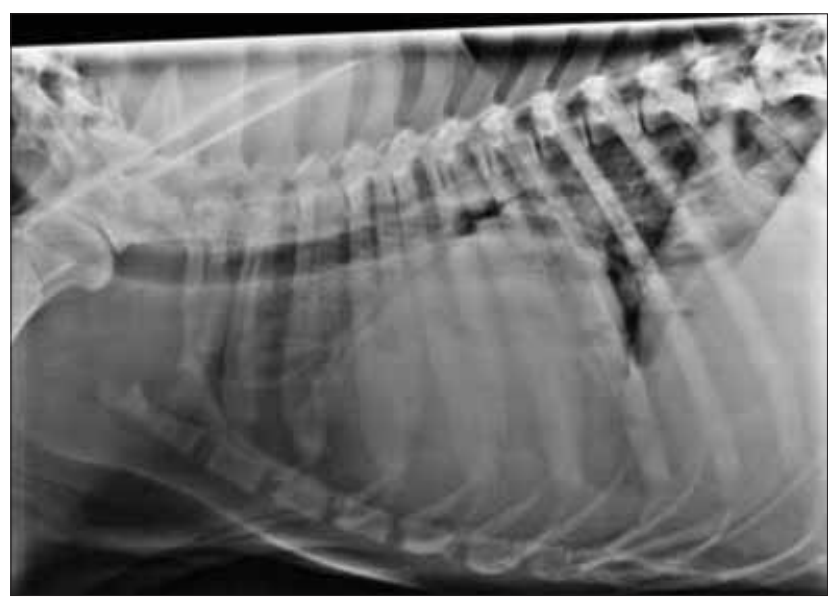

Figure-1: Lateral view of radiograph of animal affected with Dialated Cardiomyopathy. ventricular enlargement), widening of QRS complex (left ventricular enlargement) (Figure-2) depicting cardiomyopathy. It was found that in all the cases, the fractional shortening (FS\%) was less than the normal (Figure-3) and the LA/AO was more than the normal values with average of $16.50 \pm 3.70$ and $2.084 \pm 0.17$ respectively. The average of $\mathrm{LA}(4.52 \pm 0.82)$ and aorta $(2.17 \pm 0.36)$ also came out to be more than the normal values. Similarly marked increase in the average value of LVIDd (5.88 \pm 1.38$)$, and LVIDs (4.90 \pm 1.16$)$ was seen denoting dilation of the left ventricle. The E-point to septal wall separation (EPSS) was increased in four cases (Figure-4) and was normal in one case. On applying color Doppler it was seen that all the cases had mild to severe mitral regurgitation with or without the presence of aortic regurgitation (Figure-5). Regurgitation was recognized by turbulent flow of blood, which appeared as aliasing of different colors other than blue and red when Doppler was applied.

\section{PE}

One female dog of Labrador breed having PE was observed with history of lethargy, abdominal distention, exercise intolerance, coughing and subcutaneous edema. The age and body weight of the subject was 8 years and $25 \mathrm{~kg}$. The animal had tachypnea and tachycardia with respitation rate and heart rate of 30/min and 175 beats/min. On auscultation, heart sounds were slightly reduced in intensities. On abdominal palpation, ascitis was observed. The hemoglobin was 11.2 (g/dL) with white blood cell count of 9.2 (103/cumm) having $80 \%$ neutrophlils. The serum cholesterol level (151 mg/dL) was found out to be well within normal range. Ventro-dorsal radiograph of chest revealed, globoid shaped heart occupying more than $80 \%$ of the chest cavity. Cardiac silhouette was lost. Lateral view of the chest showed bilateral cardiac enlargement, pulmonary blood vessel congestion and elevated trachea (Figure-6). Electrocardiogram revealed changes like tachycardia, elevation of the ST segment, sloping of the T-wave, normal P-wave and QRS complex with no arrhythmia. But the variation in the height of the R-wave was seen indicating electrical alternans as found in PE. On echocardiography, B mode showed, a hypoechoic column around the heart depicting the fluid around the heart. When measured, the hypoechoic column of fluid was more than $1 \mathrm{~cm}$ suggesting of PE (Figure-7). The ventricular dimension was in normal range along with normal FS. EPSS was also well within the normal range. Heart was seen floating in the fluid, or we can say swinging motion of the heart. Color Doppler when applied at the left parasternal apical view, showed mild mitral regurgitation (Figure-8) while severe regurgitation at the level of aortic valves was observed.

\section{Combined DCM and PE}

Two cases, both males of each Labrador and Dalmation breed having age and body weight of 5 , 7.5 years and 25, $42 \mathrm{~kg}$ respectively have been diagnosed of having combined DCM and PE. Both had 


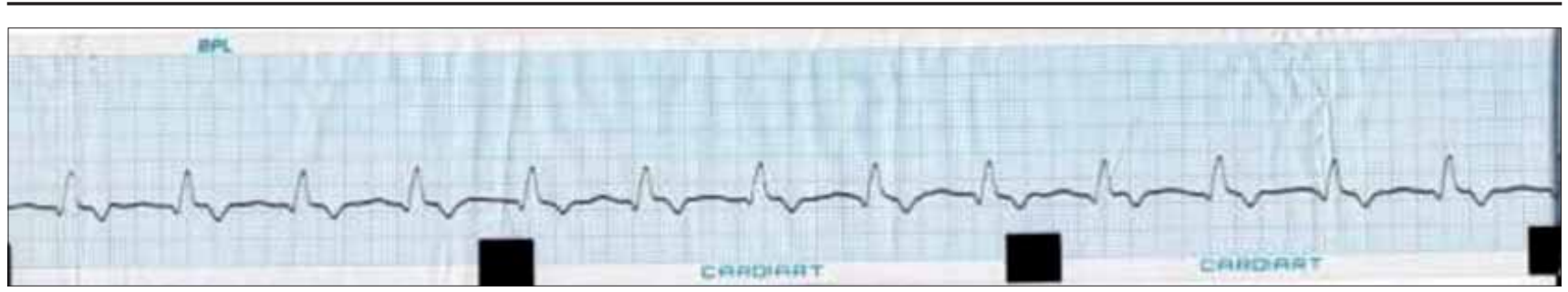

Figure-2: Electrocardiogram of animal affected with dialated cardiomyopathy.

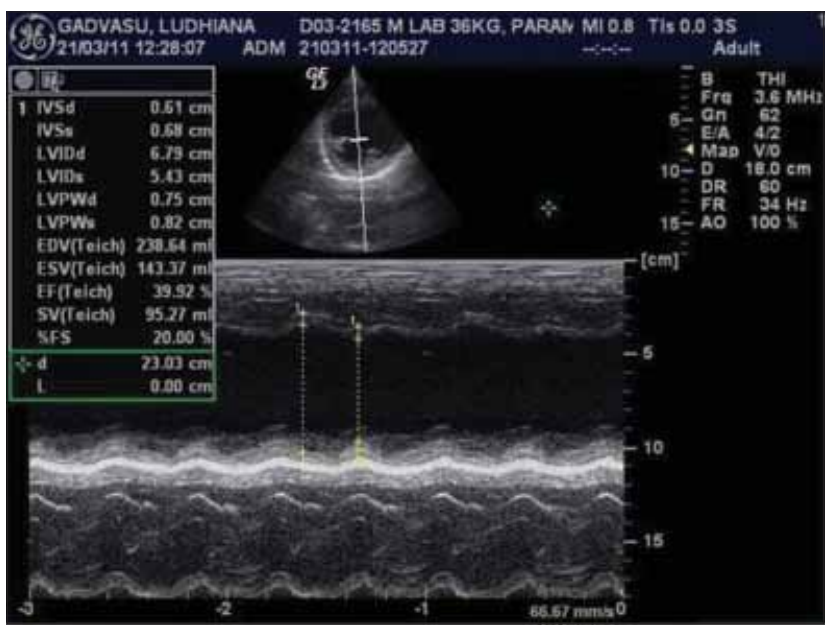

Figure-3: Decreased fractional shortening (FS\%) of the animal Affected with DCM.

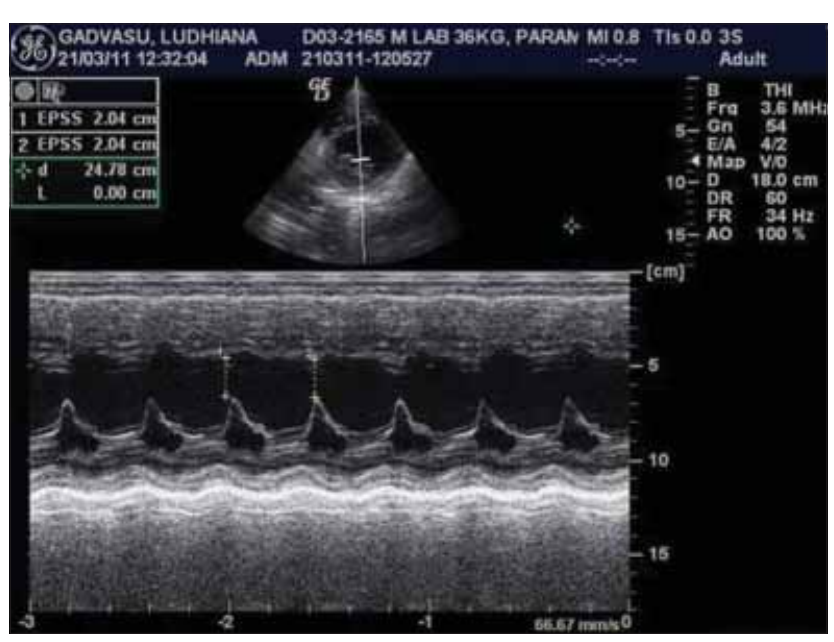

Figure-4: Increased EPSS of the animal affected with DCM.

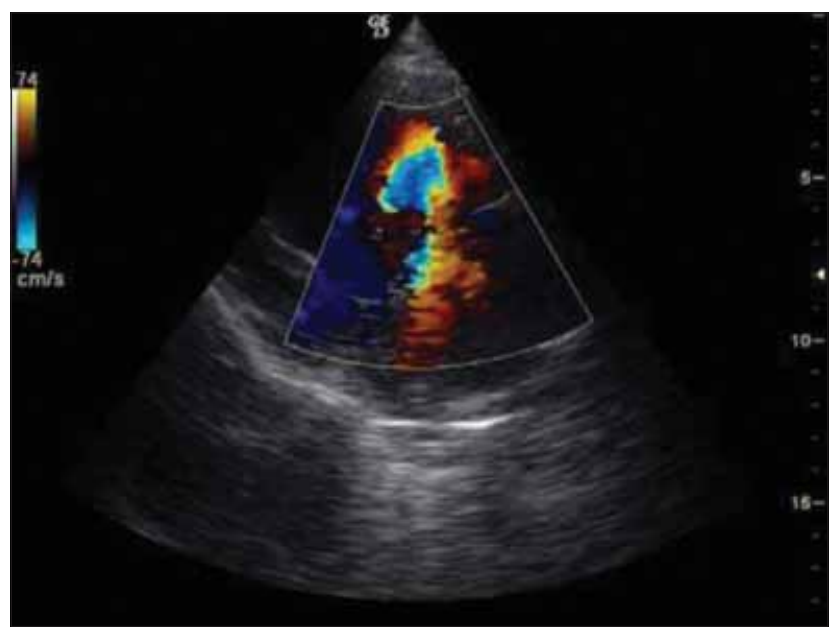

Figure-5: Regurgitation at the level of aortic valve.

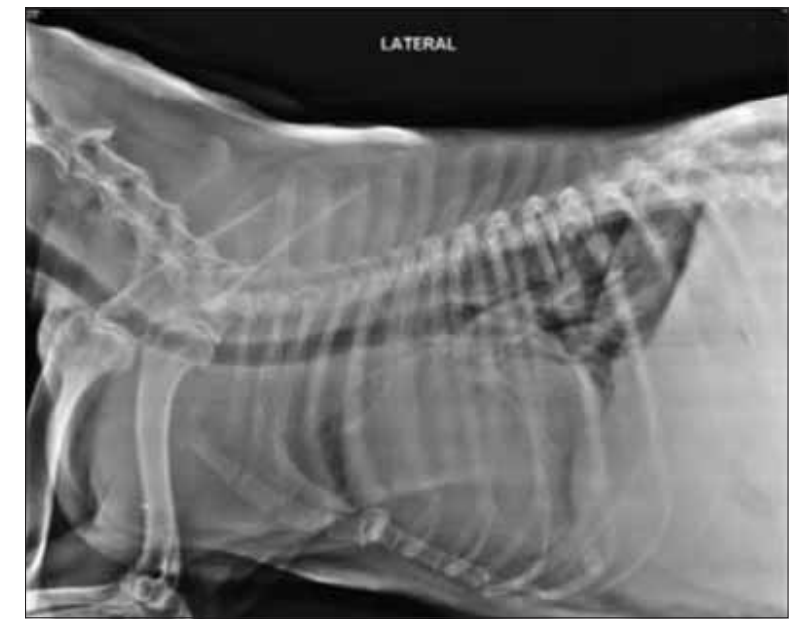

Figure-6: Lateral view radiograph of animal affected with pericardial effusion.

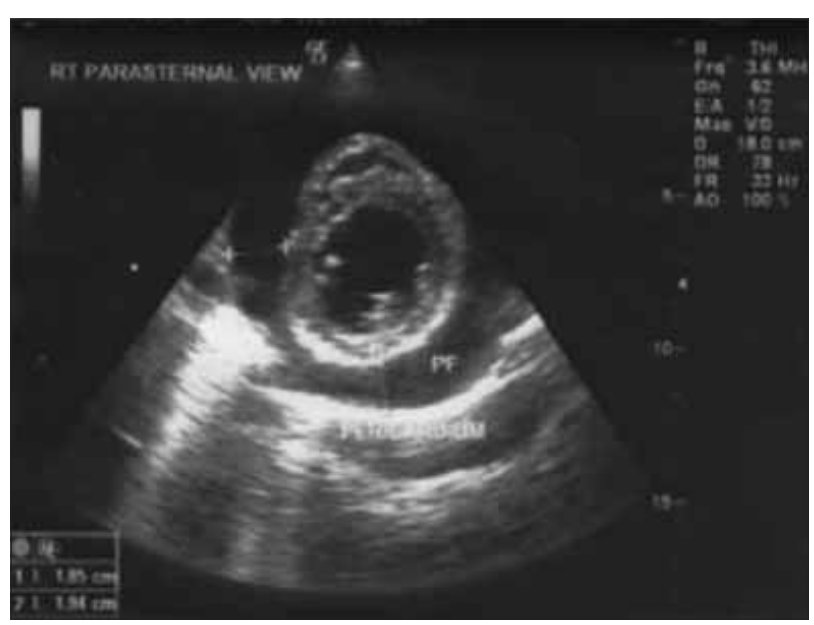

Figure-7: Anechoic column below the heart depicting increased volume of pericardial fluid.

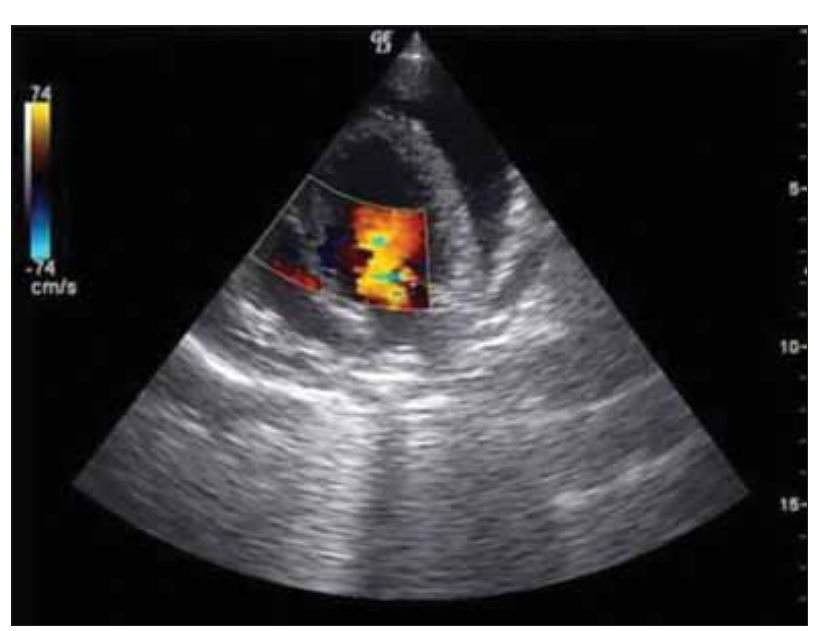

Figure-8: Regurgitation at the level of mitral valve. 
a history of subcutaneous edema and abdominal distention (ascitis), with lethargy and exercise intolerance. One dog had a history of dyspnea and syncope. The animals were tachycardic with average heart rate of 150 beats/min. One had a labored breathing and the other had tachypnea. On auscultation, muffling of heart sounds was heard. One animal was anemic with Hb 5.1 $\mathrm{g} / \mathrm{dL}$, and other had a $\mathrm{Hb}$ of $10.9 \mathrm{~g} / \mathrm{dL}$ that too on the lower side. The white blood cell count was on a higher side with an average of 14.7 (103/cumm) with 86\% neutrophilia. Lateral and ventro-dorsal views of both the subjects were suggestive of having fluid density in chest cavity and generalized cardiomegaly (Figure-9). Electrocardiogram revealed changes from normal like widening and notching of P-wave, widening of QRS complex, with or without alterance of R-wave height. Echocardiogram was also suggestive of heart floating in the chest cavity, a column of anechoic medium around heart. When further measurements were taken, it was found that the FS (Figure-10) of both the dogs was decreased and EPSS (Figure-11) was increased from the normal value. One dog had FS within normal range it could be because of early diagnosis. LA/AO ratio was increased, and a pericardial fluid column was seen around epicardium. Color Doppler studies revealed moderate to severe mitral regurgitation in both the cases.

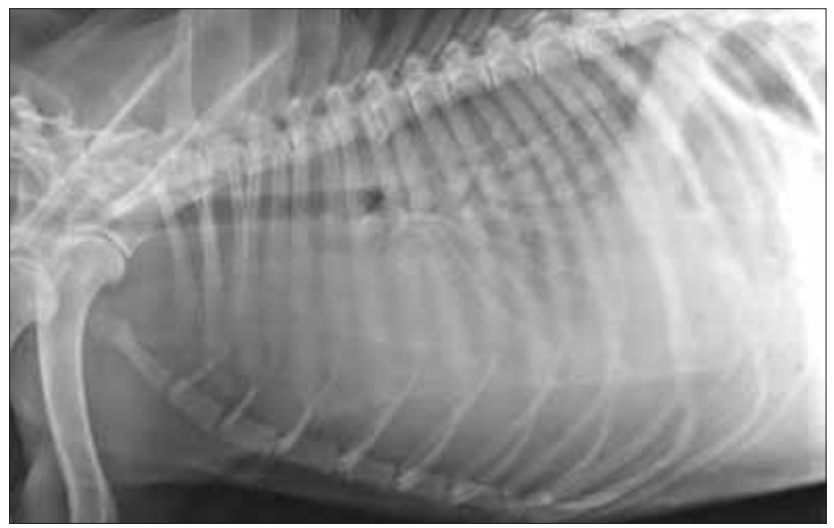

Figure-9: Lateral view radiograph of animal affected with both DCM and PE.

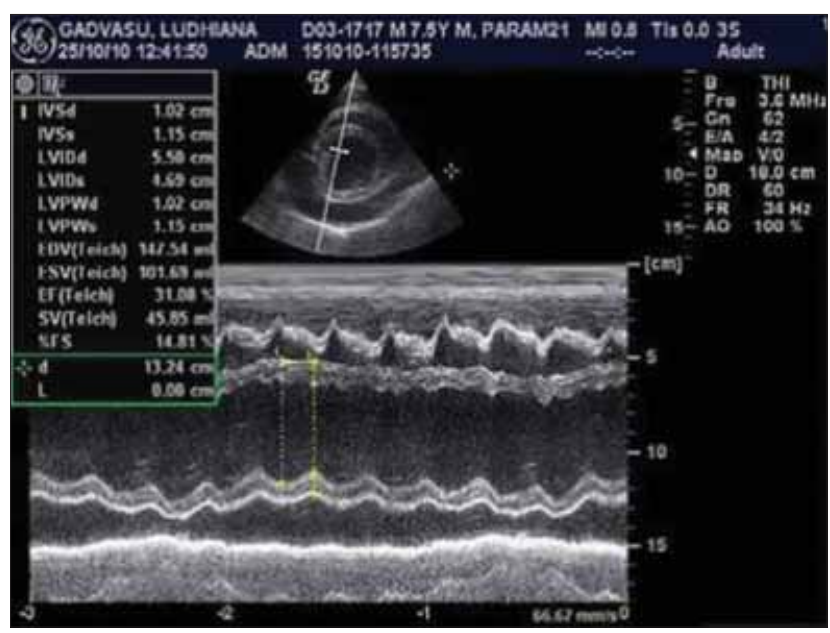

Figure-10: Decreased FS\% of animal affected with combined DCM and PE.
Abnormal on radiographic or electrocardiographic examination, but found normal on echocardiographic examination

Two cases, one each of Labrador breed (female) and Spitz breed (male) having age 10 year and 14.5 years respectively. Both dogs had tumor; Labrador had tumorous growth on rump region and Spitz had an abdominal tumor. The body weight of Labrador and Spitz was $35 \mathrm{~kg}$ and $10 \mathrm{~kg}$ respectively. Labrador had labored breathing and ascitis. But on auscultation it was normal. Heart rate was on normal with no arrhythmia. On the other hand, Spitz had continuous murmurs. Both were anemic with $\mathrm{Hb}$ of 6.1 and $6.2 \mathrm{~g} / \mathrm{dL}$ for Labrador and Spitz respectively. Both had a high white blood cell count averaging around $24\left(10^{3} / \mathrm{cumm}\right)$ with $97 \%$ neutrophils. Radiograph of one case and electrocardiogram of the other case came out to be abnormal. Lateral radiograph of Labrador was indicative of right atrial enlargement signified by J-shaped trachea. Heart enlargement was seen as the heart was occupying more than $65 \%$ of the chest cavity (Figure-12). Electrocardiogram of this dog was

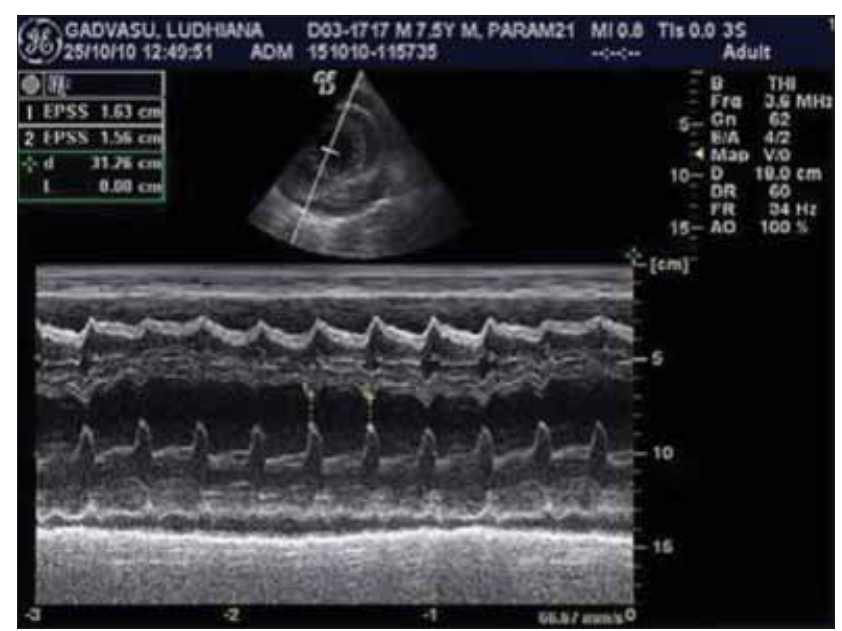

Figure-11: Increased EPSS of animal affected with combined DCM and PE.

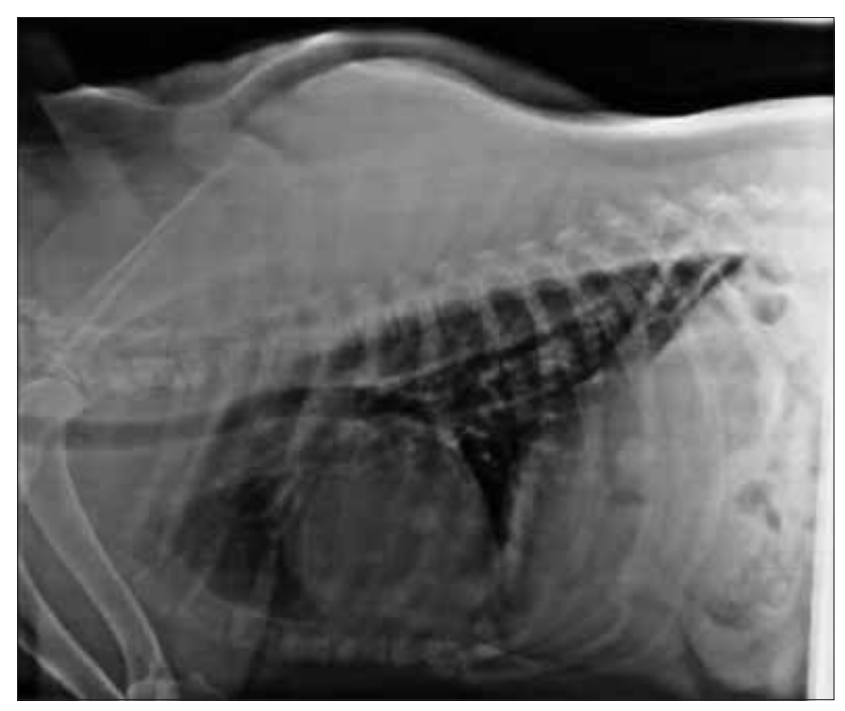

Figure-12: Lateral view radiograph of animal showing cardiomegaly. 


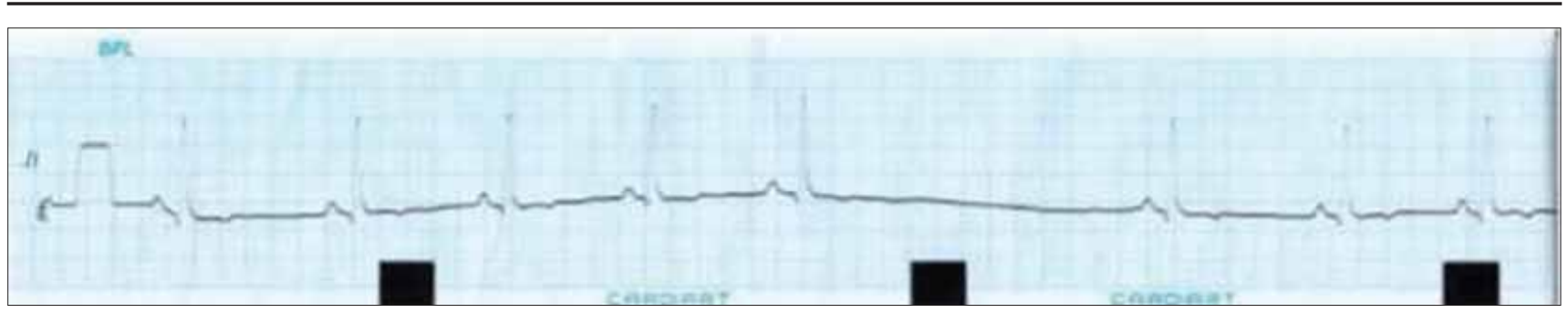

Figure-13: Electrocardiogram of animal depicting second degree AV block.

normal. Radiograph of Spitz was normal while electrocardiogram revealed second degree atrioventricular block (Figure-13). This was depicted by distance between two R-R waves more than distance between last three waves. On echocardiographic examination, all the parameters came out to be in normal range. The left ventricular dimensions, FS (Figure-14), EPSS, LA/AO ratio, all came out to be normal. Color Doppler studies (Figure-15) also revealed the same result; there wasn't any regurgitation and hence implicated the heart to be normal.

\section{Discussion}

In the cases of DCM, the FS\% was less than the normal and the LA/AO was more than the normal values with an average of $16.50 \pm 3.70$ and $2.084 \pm 0.17$ respectively. These findings were in accordance to the finding observed by other authors $[7,8]$. FS is the most common echocardiography parameter performed to see the myocardium contractility and to estimate the capability of the left ventricles $[9,10]$. Meanwhile, according to another author [11], FS is widely used as an indicator of left ventricular systolic function, and the value of $<0.25$ is usually associated with heart disease, i.e., hypovolemia. FS is also an important parameter to distinguish between hypertrophic and DCM [12]. Measurement of aortic dimensions was carried out to see abnormalities of the heart stroke volume, which decreased lumen dimension of the aorta is an indication of a low stroke volume [13]. This showed that there was dilation of the atrium size. Similar increase in atrium size due to increasing in end systolic volume was found by Calvert et al. [14]. The value of ratio $\mathrm{LA} / \mathrm{AO}$ will increase if there is dilatation of the LA [13]. Increase in the ratio of LA/ AO may also indicate the existence of left ventricular dilatation [9]. The EPSS value is usually measured to see any indication of left ventricular dilatation. When the value is more than $6 \mathrm{~mm}$, it indicates a dilatation of the left ventricle [13]. EPSS value will increase in animals with the poor myocardium contractility [9]. In the case of PE, B mode echocardiography revealed, a hypoechoic column around the heart depicting the fluid around the heart. Some authors [15] analyzed three cases having PE, the abnormalities were echofree separation of the visceral and parietal pericardium, dampening of parietal pericardial motion, exaggerated or paradoxic motion of the intracardiac structures, and thickened epicardial echoes. When measured,

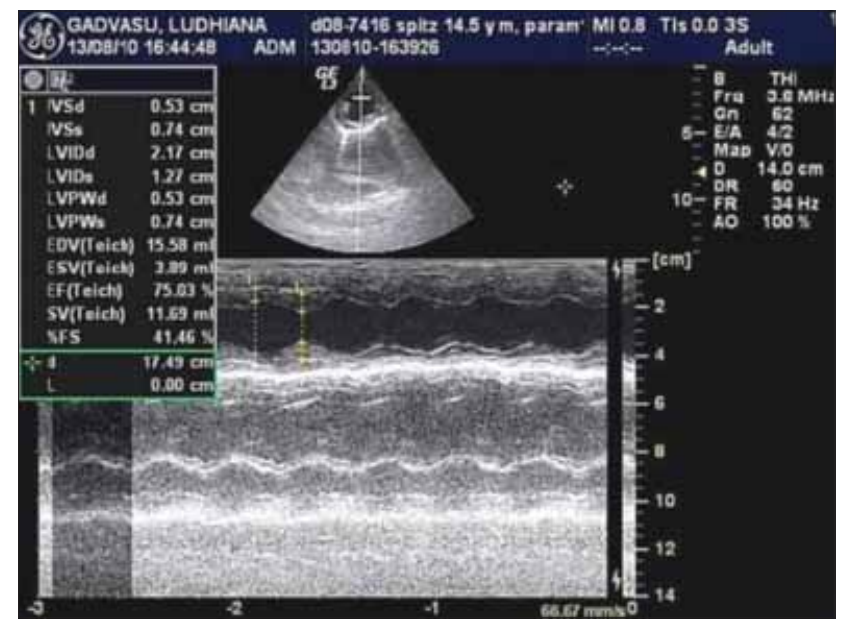

Figure-14: Normal left ventricular measurements and normal functioning of the heart.

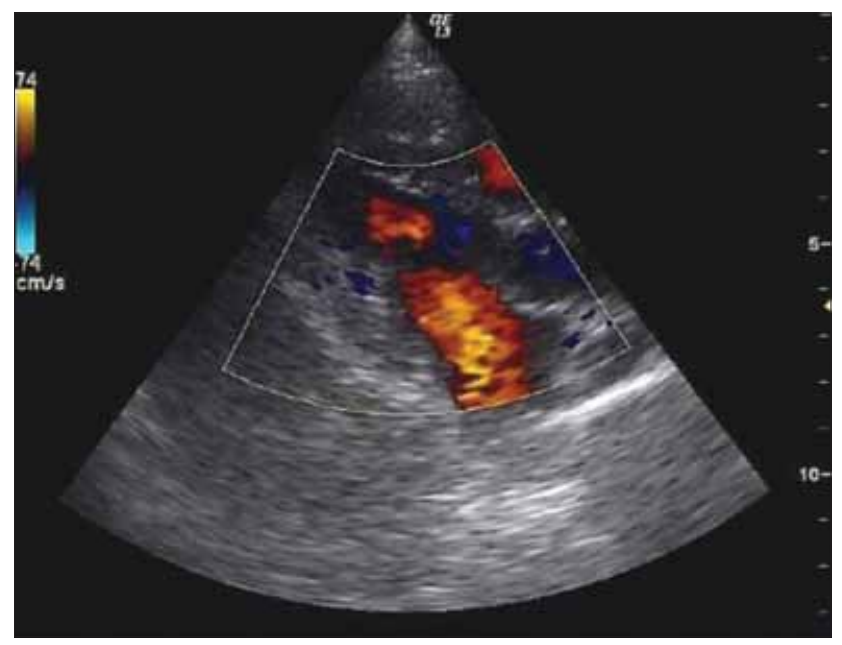

Figure-15: Normal colour doppler with no regurgitation of blood flow.

the hypoechoic column of fluid was more than $1 \mathrm{~cm}$ suggesting of PE. The ventricular dimension was in normal range along with normal FS. EPSS was also well within the normal range. Heart was seen floating in the fluid, or we can say swinging motion of the heart. This finding was also in accordance to authors $[16,17]$. Color Doppler when applied at the left parastenal apical view, showed mild mitral regurgitation while severe regurgitation at the level of aortic valves was observed. Echocardiogram of combined DCM and PE was suggestive of heart floating in the chest cavity, a column of anechoic medium around heart. When further measurements were taken, it was found that the FS of both the dogs was decreased, and EPSS 
was increased from the normal value. Color Doppler studies revealed moderate to severe mitral regurgitation. On echocardiographic examination of cases showing abnormality either on radiographic or electrocardiographic examination, all the parameters came out to be in normal range $[6,18]$. The left ventricular dimensions, FS, EPSS, LA/AO ratio, all came out to be normal. Colour Doppler studies also revealed the similar result, there wasn't any kind of regurgitation and hence implicating that the function of the heart to be normal and the changes seen in radiography and on electrocardiogram may either structural changes or electrolyte imbalance respectively.

\section{Conclusion}

Echocardiography found out to be an effective tool in canine heart disease diagnosis as it revealed the functioning of the heart.

\section{Authors' Contributions}

P Singh carried out the study, drafted and revised the manuscript. N Singh designed the experiment and guided P Singh from time to time. S. K. Mahajan and T Singh helped in carrying out the study as co-advisors and participated in scientific discussion. All the authors read and approved the final manuscript.

\section{Acknowledgments}

This research was carried out at The Department of Veterinary Surgery, College of Veterinary Science, GADVASU, Ludhiana. This study is a part of research work of Parampal Singh during his M.V.Sc. Thesis Program. No funding has been made by any department or agency in this research.

\section{Competing I nterests} interests.

The authors declare that they have no competing

\section{References}

1. Allen, D.G. (1982) Echocardiography as a research and clinical tool in veterinary medicine. Canadian Vet. J., 23: 313-316.

2. Lombard, C.W., Crispin, P. and Spencer, P. (1985) Correlation of radiographic, echocardiographic and electrocardiographic signs of left heart enlargement in dogs with mitral regurgitation. Vet. Rec., 26: 89-97.

3. Muzzi, R.A.L., Muzzi, L.A.L., Araujo, R.B.D. and Cherem, M. (2006) Echocardiographic indices in normal German Shepherd dogs. J. Vet. Sci., 7: 193-198.

4. Rao, V.V., Vasu, K. and Prathaban, S. (2008) Echocardiographic evaluation of dilated cardiomyopathy in dogs. Indian Vet. J., 85: 82-84.

5. Thomas, W.P., Gaber, C.E., Jacobs, G.J., Kaplan, P.M., Lombard, C.W., Moise, N.S. and Mose, B.L. (1993) Recommendation for standards in transthoracic two- dimensional echocardiography in the dog and cat. J. Vet. Intern. Med., 7: 247-252.

6. Yadav, B.M. (2011) Clinical studies on standardization of echocardiographic indices in dogs. M.V.Sc. Thesis Submitted to Guru Angad Dev Veterinary and Animal Sciences University, Ludhiana (Punjab) India.

7. Calvert, C.A. (1986) Dilated congestive cardiomyopathy in Doberman Pinschers. Compendium, 8: 417-430.

8. Singh, A., Singh, N. and Mahajan, S.K. (2012) Echocardiography as a definitive diagnostic tool for dialated cardiomyopathy in dogs. Indian J. Vet. Surg., 33(2): 94-98.

9. Nelson, R.W. and Couto, C.G. (1998) Small Animal Internal Medicine. $2^{\text {nd }}$ ed. Mosby Inc., USA.

10. Voros, K., Hetyey, C., Reiczigel, J. and Czirok, G.N. (2009) M-mode and two-dimensional echocardiographic reference values for three Hungarian dog breeds: Hungarian Vizsla, Mudi and Hungarian Greyhound. Acta. Vet. Hung., 57: 217-227.

11. Cornell, C.G., Kittleson, M.D. and Della Torre, P.K. (2004) Allometric scaling of $\mathrm{M}$ mode cardiac measurements in normal adult dogs. J. Vet. Intern. Med., 18: 311-321.

12. Borgarelli, M., Tarducci., A, Zanatta, R. and Haggstrom, J. (2007) Decreased systolic function and inadequate hypertrophy in large and small breed dogs with chronic mitral valve insufficiency. J. Vet. Intern. Med., 21: 61-67.

13. Goddard, P.J. (1995) Veterinary Ultrasonography. CAB International, UK.

14. Calvert, C.A., Jacobs, G.J., Pickus, C.W. and Brown, J. (1997) Signalment, survival, and prognostic factors in Doberman pinschers with end-stage cardiomyopathy. J. Vet. Intern. Med., 11: 323-326.

15. Bonagura, J.D. and Pipers, F.S. (1981) Echocardiographic features of pericardial effusion in dogs. J. Am. Vet. Med. Assoc., 179: 49-56.

16. Freestone, J.F., Thomas W.P., Carlson, G.P. and Brumbaugh GW. (1987) Idiopathic effusive pericarditis with tamponade in the horse. Equine Vet. J, 19: 38-42.

17. Boon, J. (2011) Evaluation of size, function and hemodynamics. In: Boon, J., editor. Veterinary Echocardiography. $2^{\text {nd }}$ ed. Willey-Blackwell, Ames. p153-266.

18. Singh, P. (2011) Echocardiography and Doppler studies of canine heart in health and disease. M.V.Sc. Thesis Submitted to Guru Angad Dev Veterinary and Animal Sciences University, Ludhiana (Punjab) India. 